\title{
Hybrid three-stage repair of mega-aortic syndrome with the Lupiae technique: 10-year results
}

\author{
Giampiero Esposito ${ }^{1}$, Giangiuseppe Cappabianca ${ }^{2}$, Cesare Beghi ${ }^{2}$, Antonio M. Cricco ${ }^{1}$, Cataldo Memmola ${ }^{1}$, \\ Samuele Bichi ${ }^{3}$, Matteo Miccoli ${ }^{1}$, Massimiliano Conte ${ }^{1}$, Gaetano Contegiacomo ${ }^{1}$ \\ ${ }^{1}$ Department of Cardiovascular Diseases, Mater Dei Hospital, Bari, Italy; ${ }^{2}$ Cardiac Surgery Research Centre, Insubria University, Circolo Hospital, \\ Varese, Italy; ${ }^{3}$ Department of Cardiac Surgery, Papa Giovanni XXIII Hospital, Bergamo, Italy \\ Correspondence to: Giampiero Esposito. Director, Department of Cardiovascular Diseases, Mater Dei Hospital, Bari, Italy. \\ Email: espositogp@hotmail.com.
}

\begin{abstract}
Background: Mega-aortic syndrome (MAS) is a rare disease carrying a poor prognosis if treated conservatively. Open repairs of these aneurysms are invasive, while totally endovascular repairs are associated with higher rates of late reintervention due to endoleaks, compromising long-term prognosis. We describe the 10-year results of a hybrid three-stage approach to MAS using the Lupiae technique.

Methods: Between 2006 and 2016, 27 patients with MAS extending from the ascending aorta to the iliac arteries (MAS type III) underwent: (I) a surgical aortic arch debranching, using the Vascutek Lupiae ${ }^{\mathrm{TM}}$ multibranched graft to create a proximal Dacron landing zone; (II) an abdominal aorta debranching to create a distal Dacron landing zone and (III) the implantation of multiple endovascular stents to exclude any residual aneurysm between the two landing zones.

Results: One patient died following the first stage, and another following the second stage of the repair (overall mortality 7.4\%). The interval between the first and the second stage was $58.3 \pm 16.1$ days. The interval between the second and the third stage was $47.7 \pm 13.1$ days. Four-year survival was $88.6 \% \pm 6.2 \%$ while 10 -year survival was $51.7 \% \pm 17.9 \%$. One patient had a type III endoleak after the third stage that selfresolved within 6 months without intervention. No patient had type I or II endoleaks and none underwent redo procedures. Mean follow-up was $5.9 \pm 3.6$ years and completeness was $100 \%$.

Conclusions: Three-stage hybrid repairs using the Lupiae technique can be safely performed in MAS type III patients. Short intervals between the stages should mitigate the risk of rupture during the waiting periods and may enhance patient compliance, but to achieve this, the burden and the complexity of the first stage must be carefully weighted. Our strategy improves the long-term survival of these patients compared to their natural history and is less invasive than an open repair. The adoption of Dacron landing zones appears to be associated with very low rates of reintervention due to endoleaks.
\end{abstract}

Keywords: Mega-aortic syndrome (MAS); aortic debranching; hybrid repairs

Submitted Jan 26, 2018. Accepted for publication Apr 02, 2018.

doi: 10.21037/acs.2018.04.06

View this article at: http://dx.doi.org/10.21037/acs.2018.04.06

\section{Introduction}

The mega-aortic syndrome (MAS) is a relatively rare disease that causes the aneurismal transformation of the entire thoracic aorta and a variable extent of the abdominal aorta (1). As for thoraco-abdominal aortic aneurysms (TAAA), MAS is generally recognized to have a poor mid-term prognosis if untreated (2).
We previously proposed a MAS classification $(3,4)$ according to the distal extent of the aneurysms, with MAS type I aneurysms terminating above the coeliac trunk, MAS type II aneurysms terminating between coeliac trunk and the renal arteries (RA), and MAS type III aneurysms terminating below the RA (Figure 1). The focus of this report is on MAS type III patients. 


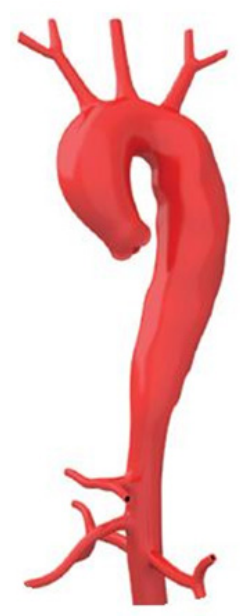

MAS type I

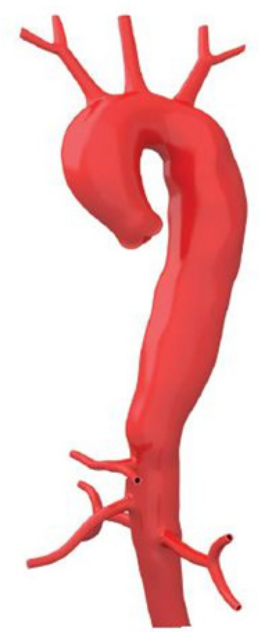

MAS type II

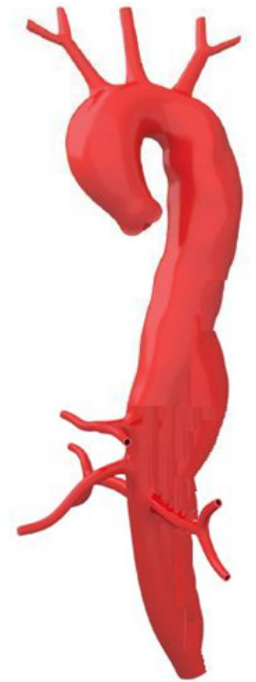

MAS type III

Figure 1 Mega-aortic syndrome (MAS) classification according to the distal extension of the aneurysm: MAS type I aneurysms extend distally above the coeliac trunk; MAS type II aneurysms extend between the coeliac trunk and the renal arteries; MAS type III aneurysms extend below the renal arteries.

In 2006, we developed a hybrid procedure called the "Lupiae technique", which is an aortic (ascending and arch) replacement using a specifically designed Dacron multibranched graft that reroutes the origin of the neck vessels close to the sino-tubular junction, creating a Dacron proximal landing zone at the level of the distal ascending aorta (5). This proximal landing zone can be used to complete the repair of a residual descending aorta aneurysm with a TEVAR. Results of this procedure for aortic dissections and thoracic aneurysms are well established $(3,4,6)$.

Nonetheless, MAS type III patients, given the distal extent of their disease, also require a visceral debranching to create a distal artificial landing zone. In this cohort, we performed a second surgical stage with an inverted Lupiae graft implanted at the level of the infra-renal abdominal aorta, rerouting close to the iliac bifurcation, the origin of the coeliac trunk, superior mesenteric artery (SMA) and RAs in order to also create a distal Dacron landing zone. A third-stage, implanting endovascular stents between the two surgically forged landing zones, completes the procedure excluding the aneurysm. The initial results of this procedure have been described here (7). We present in the current study the 10-year results of this approach.

\section{Methods}

From January 2005 to December 2016, 218 patients with multilevel aneurismal aortic disease underwent hybrid aortic repairs using the Lupiae technique. Of these, 27 patients presented with a MAS type III and underwent repair of the entire aorta with a three-stage hybrid repair. All the clinical data of these patients were retrospectively collected in a database reporting demographics, preoperative risk factors, clinical and diagnostic preoperative assessment, intraoperative features, early (30-day) and follow-up results. All patients gave their written consent to the staged procedures, which were approved by the Ethics Committee.

Preoperative imaging for elective patients included a transthoracic echocardiogram, computed tomography angiogram of the aorta with 3D reconstructions, coronary artery angiogram, and computed tomography of the neck vessels and circle of Willis to help determine whether to use unilateral or bilateral antegrade cerebral perfusion intraoperatively.

\section{Surgical and endovascular procedures}

The first and second stages were performed in the operating theatre while the final endovascular stage was performed in the angiographic suite. A scheme of the three stages is shown in Figure 2.

\section{First stage}

Totally intravenous anesthesia was routinely chosen. Left 


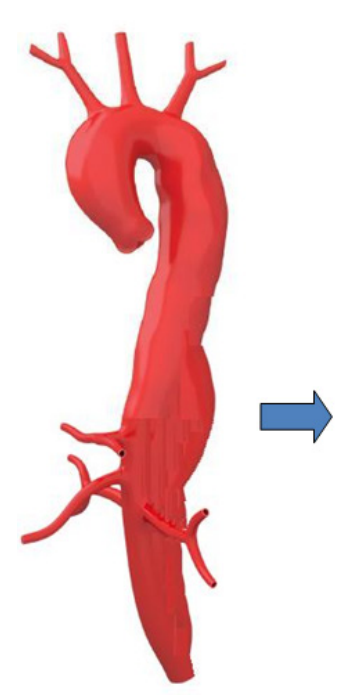

MAS type III aneurysm

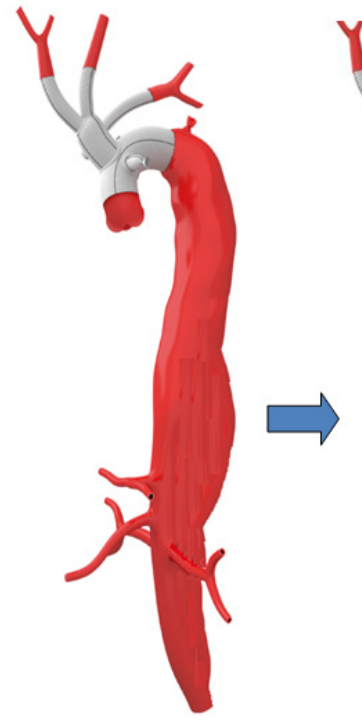

First surgical stage

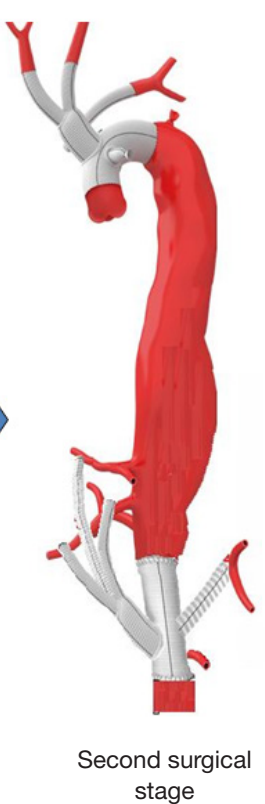

stage

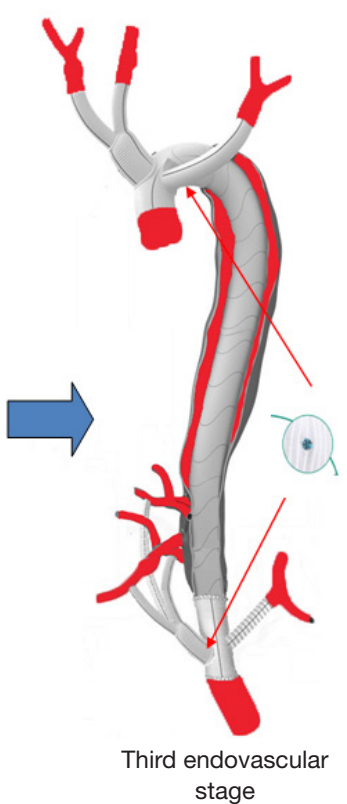

stage

Figure 2 Schematic representation of the three-stage repair in mega-aortic syndrome (MAS) type III aneurysms.

and right radial arteries were both cannulated, allowing monitoring of blood pressure during unilateral antegrade cerebral perfusion. Cerebral and, from 2014, mid-calf nearinfrared spectroscopy monitoring was used in all patients (INVOS cerebral oximeter; Somanetics Corporation, Troy, MI, USA).

A full or a partial sternotomy with a small left laterocervical incision aiming to optimize the exposure of the left subclavian artery (LSA) was performed in patients who had aneurysms involving the ascending aorta and the arch. The innominate trunk (IT), the left common carotid (LCC), and the LSA were mobilized and taped. After systemic heparinization, the IT was anastomosed end-to-side to an 8-10-mm Dacron graft that was connected to the main arterial line after the interposition of a $\mathrm{Y}$ bifurcation, used subsequently during the procedure to switch the arterial perfusion into the main Dacron graft. Before instituting cardiopulmonary bypass (CPB), the LSA was clamped, detached from the aortic arch and anastomosed end-toend to a $10-\mathrm{mm}$ Dacron graft, which was connected to a secondary perfusion line in order to achieve indirect perfusion of the upper spine and the brain through the vertebral artery.

A two-stage venous cannula was placed in the right atrium and $\mathrm{CPB}$ was instituted with anterograde perfusion through the aorta via the IT graft, whilst maintaining a core temperature of $28{ }^{\circ} \mathrm{C}$. The LSA was separately perfused at $10 \mathrm{~mL} / \mathrm{kg} / \mathrm{min}$ and the left ventricle was vented through the right superior pulmonary vein. Once the aorta was cross-clamped, antegrade cold blood cardioplegia was administered, achieving complete diastolic arrest. The aortic aneurysm was excised. If an intervention on the root or on the aortic valve was necessary, it was performed at this stage; otherwise the Lupiae graft was proximally anastomosed to the sino-tubular junction.

The Lupiae ${ }^{\mathrm{TM}}$ graft (Vascutek Terumo Inc., Scotland) presents four branches, with the first two originating from a common trunk (bovine trunk) and used to reimplant the IT and LCC. The bovine trunk and a third branch, used to reimplant the LSA, all originate from the upper margin of the graft at a narrow angle $\left(30^{\circ}\right)$, and once implanted run between the aorta and the superior vena cava, preventing branch compression once the chest is closed. A fourth branch originates from the lower margin of the graft. Alternatively, the Hemashield Platinum angled fourbranch graft (Maquet Cardiovascular LLC, USA) could be a suitable alternative to the Lupiae ${ }^{\mathrm{TM}}$ graft, given the angled take-off of the branches from the main graft.

Once the proximal anastomosis was performed, the IT was cross-clamped above the ostium, and the flow to the brain was reduced to $10 \mathrm{~mL} / \mathrm{kg} / \mathrm{min}$. The LSA perfusion was continued in parallel and at the same rate; the target 


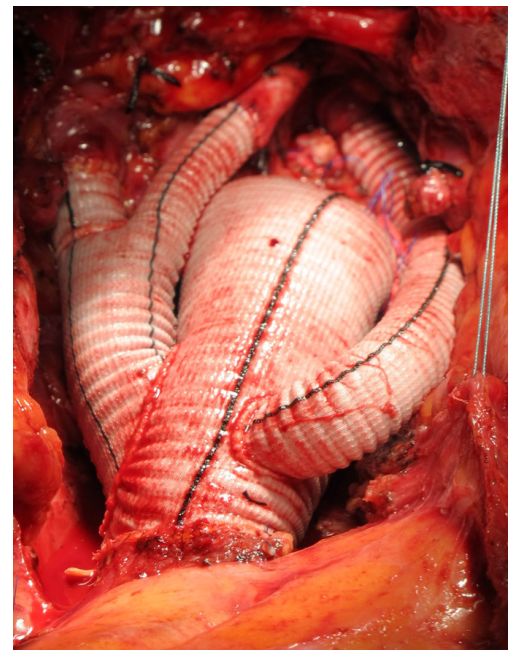

Figure 3 Surgical view of the first stage completed.

pressure for both radial arteries was $50 \mathrm{mmHg}$.

Subsequently, the cross-clamp was removed and the arch was resected between the LCC and the LSA: the distal aortic anastomosis was performed at this level. Once the distal anastomosis was completed, systemic perfusion was resumed, connecting the $\mathrm{Y}$ bifurcation previously set on the arterial line to the fourth branch of the Lupiae graft.

While the patient was rewarmed, the LCC artery and the IT were anastomosed to the two branches originating from the common trunk, and once CPB was discontinued, one of the two remaining branches was anastomosed end-to-end to the LSA (Figure 3).

\section{Second stage}

Patients underwent a midline laparotomy 4-8 weeks after the first stage. The infrarenal aorta was extensively mobilized: the iliac arteries and the RAs were taped.

The ostium of the coeliac trunk was isolated and taped at the level of the retroepiploic cavity after mobilization and twisting of the left hepatic lobe to the right was obtained, transecting the left triangular ligament. The extensive mobilization of the left renal vein allows for identification of the right and left RA, as well as the SMA in the mesocolic flexure.

After systemic heparinization, the abdominal aorta was clamped immediately below the RAs and resected down to the iliac bifurcation. If an aorto-bisiliac replacement was necessary, it was performed at this stage. The multibranched graft was implanted with the origin of the four branches close to the iliac bifurcation and pointing upward: the RAs were divided and anastomosed end-to-end to two of the four branches, and the two remaining branches were passed anterior to the pancreas and anastomosed end-toside to the coeliac trunk and end-to-end to the SMA. The ostia of the coeliac trunk and SMA were tied to prevent type II endoleaks, and the inferior mesenteric artery was reimplanted to the main Dacron graft if it was patent and did not demonstrate a satisfactory backflow once the perfusion through the coeliac trunk and SMA were restored (Figure 4).

\section{Third stage}

The endovascular stage was performed at four to eight weeks from the second stage, in the catheterisation lab and under general anesthesia with transoesophageal echocardiogram monitoring. The right femoral artery was preferred to the left, unless known to be diseased, and it was always exposed surgically. After systemic heparinization, two or three stentgrafts were deployed between the two Dacron landing zones: the origin of the branches for the neck vessels could be easily identified during the deployment of the stents, since they are marked by radiopaque dots built into the Lupiae grafts. The oversizing of the stent-grafts ranged from $5 \%$ to $10 \%$ of the aortic graft diameter (Figure 5).

\section{Follow-up}

Patients were periodically seen in the outpatient clinic, initially at three and six months and then at least once a year following the completion of the repair. Clinical evaluation and CT scans were performed. For the purpose of this study, each patient was contacted by phone in December 2017 to ascertain their clinical status. Mean follow-up was $5.9 \pm 3.6$ years ( 1.5 years minimum-12 years maximum). Follow-up completeness was $100 \%$.

\section{Statistical analysis}

Continuous variables were reported as mean \pm standard deviation, while categorical variables were presented as absolute number and percentage. The survival probability was calculated using Kaplan-Meier curves. The statistical software used was Statview 5.0.

\section{Results}

The clinical characteristics of the 27 patients with MAS type III aneurysm are described in Table 1: degenerative thoraco-abdominal aneurysm was the most common 

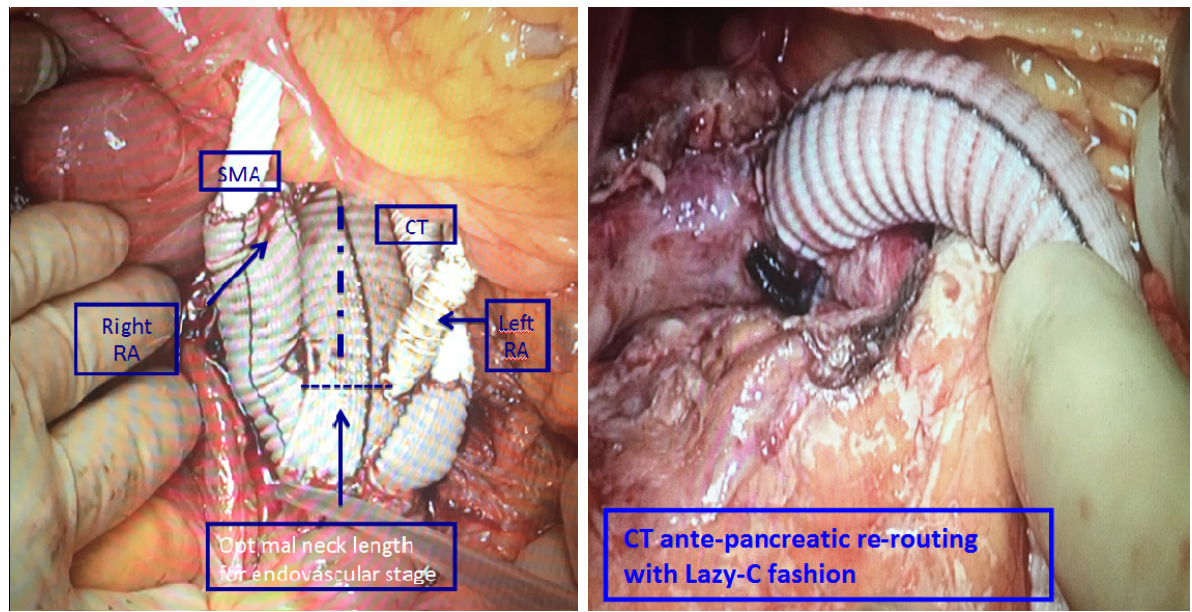

Figure 4 Surgical view of the second stage completed. On the right, the lazy C graft anastomosed on the coeliac trunk. SMA, superior mesenteric artery; CT, coeliac trunk; RA, renal artery.
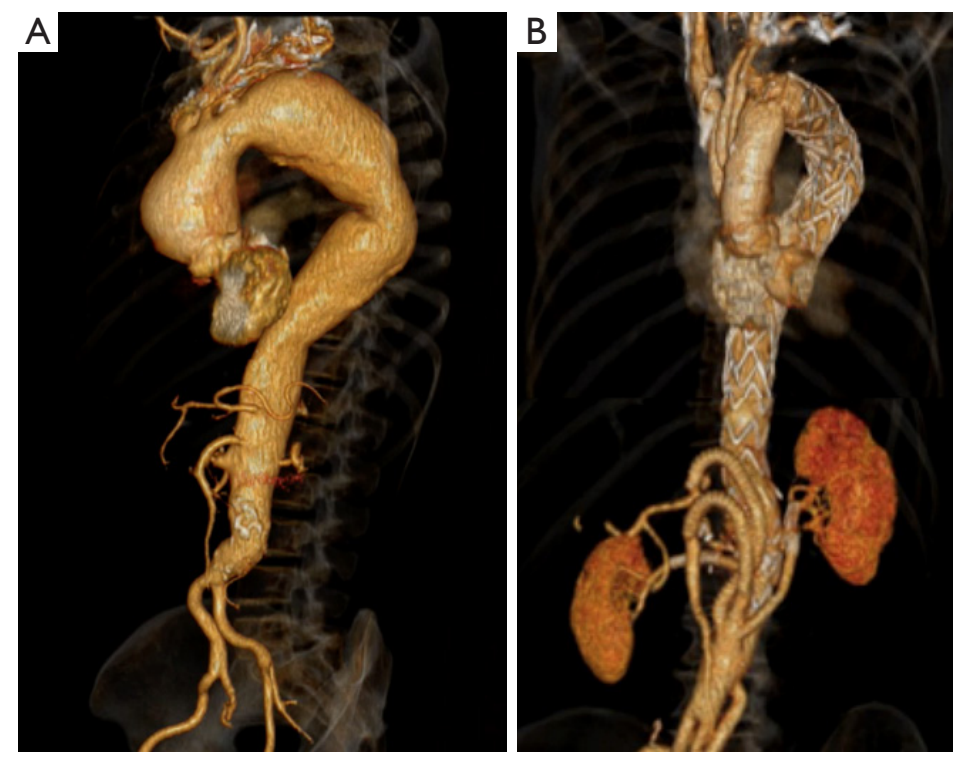

Figure 5 CT scan before (A) and after (B) the third stage of the repair. CT, computed tomography.

aetiology for MAS (70.4\%), while a minority of patients developed MAS on chronic type B dissections and only three patients presented acutely with an acute type B dissection on chronic thoraco-abdominal aneurysms: these three patients underwent the first stage urgently. Only one of these patients had a Marfan syndrome and another had previous root replacement with a Bentall procedure.

The main characteristics of the three stages of the repair are described in Table 2: three patients who did not require interventions on the aortic valve had their first surgical stage through a J-shaped mini-sternotomy. Two thirds of the patients underwent associated procedures, whereby the most commonly performed was aortic valve repair. The mean CPB temperature was $28{ }^{\circ} \mathrm{C}$ and the majority of patients underwent unilateral cerebral perfusion since the circle of Willis was functionally complete at the preoperative CT scan and a satisfactory backflow was noticed from the LCC once selective cerebral perfusion was instituted through the IT.

Only one of these patients $(3.7 \%)$ who underwent 


\begin{tabular}{|c|c|}
\hline Characteristics & Values \\
\hline Patient number & 27 \\
\hline \multicolumn{2}{|l|}{ Risk factors } \\
\hline Age, mean \pm SD (years) & $70.1 \pm 10.5$ \\
\hline Females, n (\%) & $7(25.9)$ \\
\hline Systemic hypertension, n (\%) & $23(85.2)$ \\
\hline Diabetes mellitus, n (\%) & $7(25.9)$ \\
\hline Hypercholesterolemia, n (\%) & $20(74.1)$ \\
\hline Tobacco use, n (\%) & $19(70.4)$ \\
\hline Chronic obstructive pulmonary disease, $\mathrm{n}(\%)$ & $23(85.2)$ \\
\hline Chronic renal failure, $\mathrm{n}(\%)$ & $9(33.3)$ \\
\hline History of coronary disease, n (\%) & $11(40.7)$ \\
\hline Previous cerebrovascular disease, n (\%) & $9(33.3)$ \\
\hline Obesity, n (\%) & $5(18.5)$ \\
\hline Marfan syndrome, $\mathrm{n}(\%)$ & $1(3.7)$ \\
\hline Previous cardiac surgery, n (\%) & $1(3.7)$ \\
\hline \multicolumn{2}{|l|}{ Aortic disease, n (\%) } \\
\hline Thoraco-abdominal aneurysms & $19(70.4)$ \\
\hline Chronic type B dissections & $5(18.5)$ \\
\hline $\begin{array}{l}\text { Acute type B dissections in } \\
\text { thoracoabdominal aneurysm }\end{array}$ & $3(11.1)$ \\
\hline Moderate or severe aortic valve regurgitation & $18(66.7)$ \\
\hline \multicolumn{2}{|l|}{ Aortic diameters before surgery, mean $\pm \mathrm{SD}(\mathrm{mm})$} \\
\hline Ascending aorta & $5.6 \pm 1.5$ \\
\hline Aortic arch & $5.7 \pm 1.9$ \\
\hline Descending aorta & $5.4 \pm 1.8$ \\
\hline Infrarenal aorta & $3.7 \pm 1.9$ \\
\hline
\end{tabular}

urgent repair of dissected TAAA died 60 days following the first stage due to acute distress respiratory syndrome. Four patients (14.8\%) experienced postoperative bleeding requiring re-exploration, while one patient had a slow respiratory weaning requiring tracheostomy $(3.7 \%)$. No patient experienced stroke, transient ischemic attacks, or spinal cord injuries. No patient had bowel ischemia or renal failure requiring dialysis following the first surgical stage.

Twenty-six patients underwent the second surgical stage.
Table 2 Intraoperative details $(\mathrm{n}=27)$

\begin{tabular}{ll}
\hline Details & Values \\
\hline First stage (surgical) & \\
Urgent procedure, $\mathrm{n}(\%)$ & $3(11.1)$ \\
Ministernotomy, $\mathrm{n}(\%)$ & $3(11.1)$ \\
Diameter of the Lupiae graft, mean $\pm \mathrm{SD}(\mathrm{mm})$ & $29.1 \pm 2.1$ \\
Associated procedures, $\mathrm{n}(\%)$ & $18(66.6)$ \\
Aortic valve repair & $13(48.1)$ \\
Aortic valve replacement & $3(11.1)$ \\
Bio-Bentall & $2(7.4)$ \\
Mitral valve repair & $1(3.7)$ \\
CABG & $2(7.4)$ \\
CPB data & \\
CPB temperature, mean $\pm \mathrm{SD}\left({ }^{\circ} \mathrm{C}\right)$ & $28.00 \pm 1.35$ \\
CPB time, mean $\pm \mathrm{SD}(\mathrm{min})$ & $188.2 \pm 39.9$ \\
X-clamp time, mean $\pm \mathrm{SD}(\mathrm{min})$ & $120.2 \pm 15.6$
\end{tabular}

Antegrade cerebral perfusion

Unilateral, $n(\%)$

Bilateral, n (\%)

Distal arrest time, mean \pm SD (min)

$41.5 \pm 6.1$

Second stage (surgical)

Patients, $\mathrm{n}(\%)$

$26(96.3)$

Urgent/emergency procedure, $\mathrm{n}(\%)$

$0(0)$

Interval between first and second stages, mean \pm SD (days)

$58.3 \pm 16.1$

Associated procedures

Aorto-bisiliac replacement, $\mathrm{n}$ (\%)

$10(37.0)$

Third stage (endovascular)

Patients, $\mathrm{n}(\%)$

Interval between the second and the third stage, mean \pm SD (days)

$47.7 \pm 13.1$

Mean number of stent implanted, mean $\pm S D$

$2.2 \pm 0.4$

Stent model

Gore

Valiant

Jotec-Evita

$10(37.0)$

CABG, coronary artery bypass graft; CPB, cardiopulmonary bypass; SD, standard deviation. 


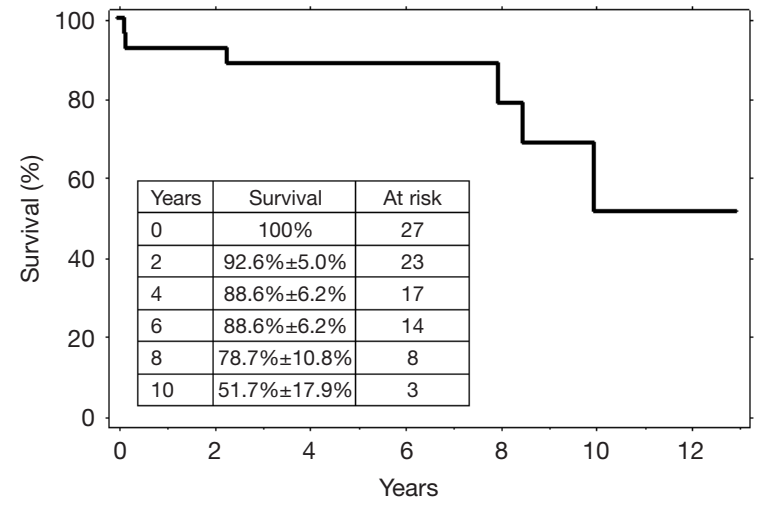

Figure 6 Ten-year survival curve for patients with mega-aortic syndrome type III aneurysm who underwent a three-stage Lupiae repair

The mean interval between the first and the second stage was $58.3 \pm 16.1$ days (range, 33.0-83.0 days). All the second stage procedures were elective and more than a third of patients had aorto-bisiliac replacement associated with abdominal aorta debranching with the inverted Lupiae graft. One patient developed bowel ischemia, underwent urgent bowel resection, and died 10 days following the procedure. Another patient had asymptomatic occlusion of the branch anastomosed to the coeliac trunk; he was managed conservatively and completed the third stage without complications.

Two patients had transient renal failure requiring hemodialysis. One patient had the rupture of the TAAA acutely following the second stage and presented with shock and hemothorax; therefore he underwent the third stage as an emergency procedure, and still survived and was able to be discharged home.

In total, 25 patients completed the third stage, undergoing implantation of the endovascular stents between the two Dacron landing zones. Apart from one patient that had emergency thoracic endovascular aneurysm repair (TEVAR), all other patients had elective procedures. The mean interval between the second and the third stage was $47.7 \pm 13.1$ days.

The mean number of endovascular stents implanted per patient was $2.2 \pm 0.4$ (19 patients had two stents, and 6 had three stents implanted). No patient died following the third stage. One patient required temporary dialysis following the third stage. All the patients underwent a computed tomography angiogram before they were discharged: one patient presented with a type III endoleak that spontaneously resolved 6 months later and the patient who underwent temporary dialysis was found to have occlusion of the graft branch anastomosed to the right RA.

\section{Follow-up}

During follow-up, four more patients died. Causes of death were: intracranial bleeding in one patient, ischemic stroke in one patient and malignancy in two patients (lung cancer in one, and bowel cancer in the other). The computed tomography angiogram performed at six months following the third stage demonstrated that the aneurysms were successfully excluded in all 25 patients. Subsequent computed tomography scans performed once a year confirmed this result and did not show any variation of the aortic diameters, or new aneurysmal or pseudoaneurysmal diseases. No patient underwent further surgical or endovascular procedures during the follow-up. The overall survival was $92.6 \% \pm 5.0 \%$ at 2 years, $88.6 \% \pm 6.2 \%$ at 6 years, and $51.7 \% \pm 17.9 \%$ at 10 years (Figure 6).

\section{Discussion}

The natural history of TAAA can be dissection or rupture, with a 5 -year survival ranging between $10-20 \%$ if left untreated (2). In this context, MAS type III is a niche disease, essentially being the retrograde extension of a Crawford type II aneurysm towards the aortic arch; it involves a minority of patients and very limited reports are available in the literature on its prevalence, treatment and outcome.

For MAS type III patients, as for Crawford type II patients, three treatments are currently available: open repair of MAS patient should require at least two stages, including a conventional elephant trunk for the proximal aorta followed by the open repair of the residual Crawford type II aneurysm. One of the largest series of Crawford type II repairs was presented by Coselli et al., including 3,309 TAAAs repairs (8), out of which 1,066 had a type II extension. For this sub-group, Coselli et al. reported an operative mortality of $9.5 \%$, cerebral complications in $11.6 \%$, spinal cord injuries in $13.9 \%$, dialysis in $9.6 \%$, tracheostomy in $12.3 \%$, and gastrointestinal complications in $4.2 \%$. Long-term survival was $63.6 \% \pm 0.9 \%$ and $36.8 \% \pm 1 \%$ at 5 and 10 years respectively (mean age at the operation: 66 years) and freedom from reoperation was $95.3 \%$ at 10 years.

The second option could be totally endovascular repair: 
for MAS type III these repairs are anecdotal, involving the use of at least two fenestrated multibranched endovascular stents for the arch and for the abdominal aorta and straight stents in between. A totally endovascular repair, even in Crawford type II patients, is still an extremely complex procedure but is less invasive than open surgery and avoids splitting the diaphragm; therefore, it should lead to a better outcome, particularly in the elderly or chronic obstructive pulmonary disease (COPD) patients.

A recent meta-analysis on totally endovascular approach for TAAAs shows a mortality rate of $9 \%$, spinal cord injury in $17 \%$ (permanent $6 \%$ ), renal failure in $15 \%$ and a reintervention rate of $21 \%$ during follow-up (9). These data are consistent with the results of a multicentre experience on 108 patients who underwent totally endovascular TAAA repair, out of which 20 had a Crawford type II aneurysm, showing a mortality rate of $9.2 \%$, spinal cord injury in $5.5 \%$ (permanent $1.8 \%$ ), and a 5 -year survival of $51 \%$, and late reinterventions in $26 \%$ (10). Both studies evidence a high rate of late reintervention, due to type I and II endoleaks, when compared to the follow-up of open repairs presented by Coselli et al.

Hybrid repair for MAS type III patients is also a complex option, involving three different procedures to complete the repair. Compared to a fully open approach, this has the advantage of being less invasive, and compared to totally endovascular repair, has the advantage of relying on Dacron landing zones, which are known to have lower rates of endoleaks and have a very limited expandability in the long term compared to native aortic tissue (11). Moreover, rerouting all the major aortic branches and oversewing the ostia of these branches on the native aorta reduces the chances of type II endoleaks. These two aspects of hybrid repairs should significantly reduce reintervention rates during the follow-up and therefore improve long-term survival.

Very limited data exist on the results of the hybrid repairs of MAS type III patients. Recently, Gkremoutis et al. (12) reported a series of 15 patients who underwent three-stage repair of MAS type III, showing an in-hospital mortality rate of $6.7 \%$, permanent paraplegia in $6.7 \%$, acute renal failure requiring temporary dialysis in $6.7 \%$, tracheostomy in $6.7 \%$, and bowel complications requiring a laparotomy in $20 \%$. One-year survival was $66 \%$ and 5 -year survival was $27.5 \%$. Type I endoleaks requiring additional stenting were reported in $6.7 \%$, while type II endoleaks occurred in $26.7 \%$.

The main difference between our technique and the one described by Gkremoutis et al. is the use of frozen elephant trunk in the first stage rather than aortic debranching with the Lupiae graft, as well as a prolonged interval between the first and second stage (average: 23 months $v s .58$ days). For the second stage, Gkremoutis et al. mentioned the use of hybrid grafts implanted in an unspecified proportion of procedures to avoid the complete dissection of visceral vessels, frequency which were not used in our series (12).

Currently, our series is not only the largest series of patients having undergone a three-stage hybrid repair for MAS type III, but also the one with the longest followup. Given the small sizes of both series, it is difficult to make reliable comparisons in terms of early mortality and morbidity, which may appear slightly lower in our series. A considerable difference can be noticed in terms of longterm survival, despite the older mean age of our patients (52.9 vs. 70.1 years). Furthermore, the frequency and the type of endoleaks reported in both series are noticeably different.

In Gkremoutis series, the choice to adopt frozen elephant trunk for the first stage, a hybrid procedure on its own, may have an impact on the burden of the first stage, as shown by longer cross-clamp time and distal arrest time possibly due to the time required to deploy the frozen elephant trunk (FET) stent. Shortening and simplifying the first stage of a three-stage procedure may have had an impact on the recovery time and the length of the interval between the first and the second stage.

The major limitation of our study is the small number of patients who underwent the three-stage procedure. Nevertheless, our data suggest that a three-stage repair with the Lupiae technique in MAS type III patients can achieve outstanding long-term results in terms of survival and freedom from reoperation, conjugating the advantages of open surgery to those of endovascular procedures.

\section{Acknowledgements}

None.

\section{Footnote}

Conflicts of Interest: The authors have no conflicts of interest to declare.

\section{References}

1. Cullhed I, Lodin H, Malers E. Mega-aorta in a young 
man. Am Heart J 1960;60:128-33.

2. Frederick JR, Woo YJ. Thoracoabdominal aortic aneurysm. Ann Cardiothorac Surg 2012;1:277-85.

3. Esposito G, Cappabianca G, Contegiacomo G, et al. Hybrid repair of thoracic and thoracoabdominal aortic aneurysms (mega aortic syndrome) with lupiae technique. Innovations (Phila) 2011;6:366-72.

4. Esposito G, Pennesi M, Bichi S, et al. Hybrid multistep approach to mega-aortic syndrome: the Lupiae technique. Eur J Cardiothorac Surg 2015;47:126-33; discussion 133.

5. Esposito G, Marullo AG, Pennetta AR, et al. Hybrid treatment of thoracoabdominal aortic aneurysms with the use of a new prosthesis. Ann Thorac Surg 2008;85:1443-5.

6. Esposito G, Cappabianca G, Bichi S, et al. Hybrid repair of type $\mathrm{A}$ acute aortic dissections with the Lupiae technique: ten-year results. J Thorac Cardiovasc Surg 2015;149:S99-104.

7. Troisi N, Bichi S, Patrini D, et al. Hybrid three-stage repair of mega aorta syndrome with the Lupiae technique. J Thorac Cardiovasc Surg 2013;145:S171-7.

Cite this article as: Esposito G, Cappabianca G, Beghi C, Cricco AM, Memmola C, Bichi S, Miccoli M, Conte M, Contegiacomo G. Hybrid three-stage repair of megaaortic syndrome with the Lupiae technique: 10-year results. Ann Cardiothorac Surg 2018;7(3):357-365. doi: 10.21037/ acs.2018.04.06
8. Coselli JS, LeMaire SA, Preventza O, et al. Outcomes of 3309 thoracoabdominal aortic aneurysm repairs. J Thorac Cardiovasc Surg 2016;151:1323-37.

9. Hu Z, Li Y, Peng R, et al. Multibranched Stent-Grafts for the Treatment of Thoracoabdominal Aortic Aneurysms: A Systematic Review and Meta-analysis. J Endovasc Ther 2016;23:626-33.

10. Youssef M, Deglise S, Szopinski P, et al. A Multicenter Experience With a New Fenestrated-Branched Device for Endovascular Repair of Thoracoabdominal Aortic Aneurysms. J Endovasc Ther 2018;25:209-19.

11. Ganapathi AM, Andersen ND, Hanna JM,et al. Comparison of attachment site endoleak rates in Dacron versus native aorta landing zones after thoracic endovascular aortic repair. J Vasc Surg 2014;59:921-9.

12. Gkremoutis A, Zierer A, Schmitz-Rixen T, et al. Staged treatment of mega aortic syndrome using the frozen elephant trunk and hybrid thoracoabdominal repair. J Thorac Cardiovasc Surg 2017;154:1842-9. 